REVIEW ARTICLE

PRACA POGLĄDOWA

\title{
PRIORITIES OF ANTI-HYPERGLYCAEMIC DRUG THERAPY IN PATIENTS WITH TYPE 2 DIABETES AND HEART FAILURE
}

DOI: 10.36740/WLek202003139

\author{
Maryana M. Rosul, Miroslava M. Bletskan, Nataliya V. Ivano, Marina 0. Korabelschykova, Yelyzaveta I. Rubtsova
}

STATE HIGHER EDUCATIONAL ESTABLISHMENT “UZHHOROD NATIONAL UNIVERSITY”, UZHHOROD, UKRAINE

\begin{abstract}
The aim is to explore the possibilities of improving the effectiveness in preventing cardiovascular diseases and heart failure using sodium-glucose co-transporter 2 inhibitors. Materials and methods: The analysis of the existing clinical and experimental data on the effect of sodium-glucose co-transporter 2 (SGLT-2) inhibitors on the cardiovascular system, the condition of kidneys, cardiovascular risk factors.

Review: SGLT-2 inhibitors are the first class of glucose-lowering agents in large-scale studies (EMPA-REG OUTCOME, CANVAS, CVD-REAL, CVD-REAL2) which have demonstrated the ability to improve cardiorenal outcomes and reduce the risk of hospitalization with heart failure in patients with diabetes. In addition to hypoglycaemic action, SGLT-2 inhibitors show a number of pleiotropic effects, which are potentially capable of reducing cardiovascular risk: diuretic effect, decrease in: blood pressure, arterial wall stiffness, waist and body weight, expression of albuminuria, etc. The use of drugs of this class opens great prospects not only in terms of glycaemic control, but also in the prevention of cardiovascular complications of diabetes.

Conclusions: 1 . When choosing glucose-lowering agents in patients with type 2 diabetes, it is necessary to take into account their impact on the risk of development and the course of heart failure.

2. SGLT-2 inhibitors ought to be considered as a preferred method of treatment for type 2 diabetes in patients with heart failure or with a risk of heart failure that meets the latest recommendations of the European and American Diabetes Association.
\end{abstract}

KEY WORDS: type 2 diabetes; heart failure, sodium-glucose co-transporter 2 inhibitors

Wiad Lek. 2020;73(3):609-613

\section{INTRODUCTION}

Nowadays cardiovascular pathology and diabetes represent a significant medical and social problem due to the serious consequences for health, work efficiency, life expectancy and life quality of patients [1]. Diabetes mellitus is an independent risk factor for cardiovascular diseases. At the same time, cardiovascular complications are the major cause of death in patients with type 2 diabetes [2]. At the same time, the risk of developing heart failure in patients with type 2 diabetes increases 2- 4 times $[3,4]$. This leads to a significant increase in the frequency of hospitalization and a pronounced deterioration in prognosis: 5 -year survival in comorbidity of heart failure and type 2 diabetes does not exceed $25 \%[5,6,7]$. The urgent task is to look for preventative measures of heart failure in patients with type 2 diabetes. It includes the improvement of glucose-lowering therapy, namely the use of drugs that do not only improve glycaemic control but also reduce the risk of cardiovascular diseases and heart failure. Recently, an arsenal of agents for the treatment of type 2 diabetes has been supplemented with sodium-glucose co-transporter 2 inhibitors. They are associated with a decrease in glucose reabsorption in the proximal tubules of the kidneys, provided that the glomerular filtration rate is maintained.

\section{THE AIM}

is to study the possibilities of increasing the efficiency of prevention of cardiovascular diseases and heart failure when using a new class of glucose-lowering agents - sodium-glucose co-transporter 2 inhibitors.

\section{MATERIALS AND METHODS}

The analysis of the existing clinical and experimental data on the effect of SGLT-2 inhibitors on the cardiovascular system, kidney status, risk factors for cardiovascular complications.

\section{REVIEW AND DISCUSSION}

The study of new pathogenetic mechanisms responsible for maintaining chronic hyperglycaemia has led to the creation of a promising class of glucose-lowering agents that reduce plasma glucose by inhibiting glucose reabsorption in the proximal tubules of kidneys without hypoglycaemia [8]. Due to its mechanism of action, SGLT-2 inhibitors are not only able to improve glycaemic control but also to provide cardioprotective and nephroprotective effects in patients with type 2 diabetes and high cardiovascular risk. 
The EMPA-REG OUTCOME study evaluated the effects of oral intake of 10 and $25 \mathrm{mg}$ of empagliflosin on cardiovascular morbidity and mortality in patients with type 2 diabetes and high cardiovascular risk. The results have shown a $14 \%$ reduction in the risk of serious side cardiovascular events (severe cardiovascular event; non-fatal myocardial infarction; non-fatal stroke and mortality from cardiovascular disease), a decreased risk of cardiovascular mortality by $38 \%$ and overall mortality by $32 \%$, hospitalization frequency with heart failure by $35 \%$ on receiving empagliflozin compared with placebo [9]. A low risk of acute heart failure was observed in patients receiving empagliflozin with a history and without a history of heart failure [10]. The favorable effect of empagliflozin on reducing deaths and hospitalizations due to heart failure was common in patients throughout the observation period and after discontinuation of the drug (up to 3.1 years). Both doses of empagliflozin ( $10 \mathrm{mg}$ and $25 \mathrm{mg}$ ) had similar effects on cardiovascular outcomes, so this effect was not dose-dependent. Besides the effects on cardiovascular risk, based on additional data from EMPA REG-Outcome, it has been shown that the addition of empagliflozin to the standard therapy significantly slowed the development and progression of kidney damage in patients both without albuminuria and with microalbuminuria and had the greatest effect on patients with the most severe course of nephropathy (existing macroalbuminuria) [11].

Prior to the publication of the results of the EMPA-REG study, the ability to improve the prognosis of patients with heart failure had not been proven for any of the glucose-lowering agents, moreover, some of these agents can impair it [12].

Positive results of SGLT-2 inhibitors were also obtained in another clinical trial program, CANVAS, which examined the safety and efficacy of canagliflozin in patients with type 2 diabetes who had a history of cardiovascular events $(65 \%)$ or with a high cardiovascular risk (35\%). Adding canagliflozin to the standard therapy not only reduced blood pressure and body weight, but also reduced the rate of hospitalization for heart failure by $33 \%$. The drug also showed a nephroprotective effect due to its ability to slow albuminuria progression by $27 \%$ and increase albuminuria regression by $70 \%$. The level of an estimated glomerular filtration rate was stable in the main canagliflozin group whereas it decreased in placebo patients [13].

A large international SVD-REAL study evaluating the data of more than 300,000 diabetic patients ( $87 \%$ of patients did not have cardiovascular disease at the beginning of the study) has demonstrated that SGLT-2 inhibitors compared to other diabetes medications are associated with a 39\% relative reduction in the risk of hospitalization for heart failure and overall mortality by $51 \%$ [14]. In another CVD-REAL2 international study, the use of SGLT-2 inhibitors was associated with a $49 \%$ reduction in the risk of death and a $36 \%$ reduction in the risk of hospitalization for heart failure [15]. It should be noted that the vast majority of patients in this study did not have a diagnosis of cardiovascular disease. Therefore, the findings in the CVD-REAL and
CVD-REAL2 studies regarding the cardiovascular benefits of SGLT-2 inhibitors can be extrapolated to a broader population of patients with diabetes.

It is not yet fully understood what underlies the cardioprotective action of SGLT-2 inhibitors. These mechanisms are most likely not associated with glycaemia reduction. A number of additional effects of SGLT-2 inhibitors (the so-called pleiotropic non-hypoglycaemic effects) may be viewed on the example of empagliflozin. It is broadly accepted that patients with diabetes are known to be characterized by an excess of $\mathrm{Na}+$, due to its increased reabsorption in the kidneys by hyperglycaemia, hyperinsulinemia, activation of the renin-angiotensin-aldosterone system, etc. [16].

The delay of $\mathrm{Na}+$ and water plays an important role in increasing the preload and afterload of the heart, leading to the development of peripheral oedema and stagnation of blood in the lungs, and eventually to hospitalization. In this case, the excess of $\mathrm{Na}+$ is distributed not only in extracellular space, but also inside cells. The excessive content of $\mathrm{Na}+$ inside cardiomyocytes increases the risk of arrhythmia in the experiment and may lead to impaired myocardial function, in particular through impaired mitochondrial function [17].

Empagliflozin has the properties of an osmotic diuretic and enhances natriuresis. By reducing the volume of blood plasma, it helps to achieve euvolaemic state and lower overload on the ventricles of the heart and, consequently, reduce the volume overload of the heart, which can play a role in reducing the risk of arrhythmias and arrhythmic death. Reduction of peripheral vascular resistance and blood pressure can reduce cardiac afterload, improve coronary blood flow and myocardial contractility. Among the important additional advantages there are the lack of activation of the sympathetic nervous system and the absence of changes in potassium levels, since the occurrence of hyperkalaemia leads to a decrease in the positive effect of diuretic therapy on the frequency of cardiovascular events [18]. This may attribute empagliflozin to diuretics with unique properties, and it is likely that SGLT-2 inhibitors will be more in demand, cardiology included, than conventional diuretic therapy after further large-scale studies.

Several studies have shown that SGLT-2 inhibitors cause a decrease in systolic blood pressure in the range of 3-5 $\mathrm{mm} \mathrm{Hg}$ and diastolic blood pressure $-2-3 \mathrm{~mm} \mathrm{Hg}$, reduce the pulse pressure and the average blood pressure [19]. In particular, in the EMPA-REG Outcome study, the reduction in systolic blood pressure after empagliflozin was $4 \mathrm{~mm} \mathrm{Hg}$, while diastolic blood pressure was $2 \mathrm{~mm} \mathrm{Hg}$, which was not accompanied by an increase in heart rate. This leads to the conclusion that there is no compensatory reflex activation of the sympathetic nervous system and suggests the effect of SGLT-2 inhibition on the reduction of the stiffness of the arterial vessel wall. Thus, the use of empagliflozin in young patients with an uncomplicated course of type 1 diabetes [20] led to a decrease in systolic blood pressure (an average of $2.7 \mathrm{~mm} \mathrm{Hg}$ ). It was also noted that the drug reduced the speed of the pulsatile wave and of the radial artery. 
Therefore, reducing the incidence of cardiovascular complications and mortality in patients with type 2 diabetes compared with other diabetes-reducing agents or placebo may primarily be related to the hemodynamic effects of SGLT-2 inhibitors [21, 22, 23], which reduce preload and afterload, vascular stiffness, which improves left ventricular function and leads to a decrease in myocardial oxygen consumption.

In people with type 2 diabetes, SGLT- 2 inhibitors, in addition to the antihypertensive effect, have caused dose-dependent weight loss as a result of the osmotic diuretic effect and loss calories for glucosuria $[24,25,26]-$ excretion of 50-80 g of glucose a day stands for losing 200-300 kcal. In addition, another important aspect is a lower level of insulin, which is for its anabolic effects, as well as an increase in the oxidative metabolism of adipose tissue [27]. This results in gradual (usually within a few months) reduction of the body mass by $2-3 \mathrm{~kg}$, with the further stabilization of body weight after 3-6 months [28]. Investigating the results of EMPA-REG Outcome in the course of empagliflozin treatment, the lowering of an average body weight is pointed out by $2 \mathrm{~kg}$, resulting in a decrease in waist by $2 \mathrm{~cm}$. The influence of SGLT-2 inhibitors on visceral fat is of a special interest. The latter is associated with a higher degree of probability of type 2 diabetes development, cardio vascular complications and death [29].

Among the beneficial extraglicaemic effects of SGLT-2 inhibitors there is a glucose-dependent decrease in urate reabsorption [30]. SGLT-2 inhibitors increase the excretion of uric acid and reduce its plasma concentration by $10-15 \%$, which within the EMPA-REG OUTCOME is $24 \mu \mathrm{mol} / 1$. For a long time, hyperuricemia was not only considered as a component of the metabolic syndrome, but was also associated with the decrease in cardiovascular diseases [31]. The accumulated data, both in humans and in experimental models, illustrate that the increased plasma level of uric acid may lead to arterial hypertension, endothelial vascular dysfunction, congestive heart failure, and kidney dysfunction [32].

The decrease in uric acid levels can hardly explain the rapid improvement in cardiovascular outcomes demonstrated by empagliflozin compared to placebo. Nevertheless, this effect may play a certain role in reducing cardiovascular mortality at a later period of drug administration and slow down the progression of diabetic nephropathy.

Empagliflozin does not increase the incidence of hypoglycaemia regardless of baseline glycated hemoglobin (HbA1c) concentration, which gives an opportunity to improve cardiovascular prognosis without increasing the risk of hypoglycaemia. The second benefit is that the risk of hypoglycaemia does not increase depending on the basic therapy. It should be emphasized that the mechanism of action of SGLT-2 inhibitors is insulin-independent. Therefore, SGLT-2 inhibitors can be used at any stage of type 2 diabetes, in particular in the depletion of beta-cellular apparatus of the pancreas. Thus, they can be combined with other anti-hyperglycaemic drugs, since their mechanisms are different from the mechanisms of action of other cur- rently available classes of drugs [33]. SGLT-2 inhibitors are the only class of glucose-lowering agents, other than metformin, that are, without any limitation, compatible with basal insulin. The addition of SGLT-2 inhibitors to the therapy regimen helps to improve glycaemic control with a lower insulin dose.

Numerous data have convincingly shown that chronic kidney disease resulting from diabetic nephropathy is an important and independent risk factor for cardiovascular pathology. Population studies have shown that a combination of the chronic kidney disease and type 2 diabetes in a patient significantly increases the incidence of cardiovascular complications. However, albuminuria has also been identified as a risk factor for death from a cardiovascular disease. The analysis of a numerous randomized placebo-controlled clinical trials, including EMPA-REG OUTCOME, CANVAS, has shown that SGLT-2 inhibition can cause both cardiovascular and renoprotective effects $[34,35,36,37]$.

SGLT-2 inhibitors have been proven to prevent impaired glomerular filtration and reduce the degree of albuminuria in patients with diabetes-related kidney disease. In the Renal branch of the EMPA-REG OUTCOME study, there was a significantly lower risk of progression to macroalbuminuria or other clinically relevant renal outcomes, such as doubling of serum creatinine levels and initiation of renal replacement therapy in patients from the empagliflozin group in comparison to the placebo one. Thus, the obtained results allow us to confidently attribute empagliflozin to drugs with a nephroprotective action. The mechanism of nephroprotective action of empagliflozin, apparently, is not only due to the decrease in glycaemia, but also due to the non-glycaemic effects in the form of weight loss, decrease in blood pressure and stiffness of the arterial wall, correction of intrarenal hemodynamics, increase of natriuresis. Nephroprotection can be associated with the direct effect of empagliflozin on inflammatory processes, since inflammation, fibrosis and oxidative stress are closely related to intracellular hypertension [38]. The results of in vitro and in vivo studies suggest that inhibition of SGLT-2 slows the activity of these processes [39]. However, in studies with empagliflozin, inflammatory markers have not been evaluated, although there is some evidence that $\mathrm{C}$-reactive protein reduced dapagliflozin [40]. This will possibly be the subject of the further study. The results of studies on the prospects of using SGLT-2 inhibitors for the treatment of heart failure in individuals without diabetes will also be of great interest.

\section{CONCLUSIONS}

1. When choosing glucose-lowering agents in patients with diabetes, it is necessary to consider their impact on the risk of development and the course of heart failure.

2. SGLT-2 inhibitors should be considered as a preferred method of treatment for diabetes in patients with heart failure or with a risk of heart failure, which meets the recent recommendations of the European and American Diabetes Association. 


\section{REFERENCES}

1. Ogurtsova K, da Rocha Fernandes JD, Huang Y, et al. IDF Diabetes Atlas: Global estimates for the prevalence of diabetes for 2015 and 2040. Diab Res Clin Pract. 2017; 128: 40-50.

2. Shah A.D., Langenberg C, Rapsomaniki E et al. Type 2 diabetes and incidence of cardiovascular diseases: a cohort study in 1.9 million people. Lancet Diabetes Endocrinol. 2015; 3 (2): 105-113.

3. Kannel W., McGee D. Diabetes and cardiovascular disease: the Framingham study. JAMA. 1979; 241: 2035-2038.

4. Nichols G., Gullion C., Koro C. et al. The incidence of congestive heart failure in type 2 diabetes: an update. Diabetes Care. 2004; 27: 1879-1884.

5. Cavender M.A., Steg P.G., Smith S.C. Jr., et al. REACH Registry Investigators. Impact of diabetes mellitus on hospitalization for heart failure, cardiovascular events, and death: outcomes at 4 years from the reduction of Atherothrombosis for Continued Health (REACH) Registry. Circulation. 2015; 132 (10): 923-931.

6. Dunlay S., Redfield M., Weston S. et al. Hospitalizations after heart failure diagnosis: a community perspective. J Am Coll Cardiol. 2009; 54: 1695-1702.

7. Chaudhry S., McAvay G., Chen S. et al. Risk factors for hospital admission among older persons with newly diagnosed heart failure: findings from the Cardiovascular Health Study. J Am Coll Cardiol. 2013; 61: 635-642.

8. Washburn W.N. Evolution of sodium glucose cotransporter 2 inhibitors as anti-diabetic agents. Exp 0pin Ther Patents 2009; 19: 1485-1499.

9. Zinman B., Wanner C., Lachin J. et al. Empagliflozin, Cardiovascular Outcomes, and Mortality in Type 2 Diabetes. N Engl J Med. 2015; 373: 2117-2128

10. Fitchett D., Zinman B., Wanner C. et al.; the EMPA-REG OUTCOME Trial Investigators. Heart failure outcomes with empagliflozin in patients with type 2 diabetes at high cardiovascular risk: results of the EMPA-REG OUTCOME. Eur Heart J. 2016;37:1526-1534.

11. Wanner C, InzucchiSE, Lachin JM, et al. Empagliflozin and Progression of Kidney Disease in Type 2 Diabetes. N Engl J Med. 2016; 375(4): 323-334.

12 GilbertRE1, Krum H2. Heartfailure in diabetes: effects of anti-hyperglycaemic drug therapy. Lancet. 2015 May 23; 385 (9982): 2107-2117.

13. Neal B., Perkovic V., Mahaffey K. et al.; for the CANVAS Program Collaborative Group. Canagliflozin and cardiovascular and renal events in type 2 diabetes N Engl J Med. 2017; 377: 644-657.

14. Kosiborod M., Cavender M., Fu A. et al.; on behalf of the CVD-REAL Investigators and Study Group. Lower risk of heart failure and death in patients initiated on sodium-glucose cotransporter- 2 inhibitors versus other glucose-lowering drugs: the CVD-REAL Study (Comparative Effectiveness of Cardiovascular Outcomes in New Users of SodiumGlucose Cotransporter-2 Inhibitors). Circulation. 2017; 136: 249-259.

15. Kosiborod M., Lam C., Kohsaka S. et al.; CVD-REAL Investigators and Study Group. Cardiovascular events associated with SGLT-2 inhibitors versus other glucose-lowering drugs: the CVD-REAL 2 Study. J Am Coll Cardiol. 2018; 71: 2628-2639.

16. Heerspink HJ, Perkins BA, Fitchett DH, et al. Sodium Glucose Cotransporter 2 Inhibitors in the Treatment of Diabetes Mellitus: Cardiovascular and Kidney Effects, Potential Mechanisms, and Clinical Applications. Circulation. 2016; 134 (10): 752-772.

17. Liu T, Takimoto E, Dimaano VL, et al. Inhibiting mitochondrial $\mathrm{Na}+/ \mathrm{Ca} 2+$ exchange prevents sudden death in a Guinea pig model of heart failure. Circ Res. 2014 115(1): 44-54. 18. Franse LV, Pahor M, Di Bari M, et al. Hypokalemia Associated With Diuretic Use and Cardiovascular Events in the Systolic Hypertension in the Elderly Program. Hypertension. 2000; 35(5): 1025-1030.
19. Vasilakou D, Karagiannis T, Athanasiadou E, et al. Sodium-glucose cotransporter 2 inhibitors for type 2 diabetes: a systematic review and meta-analysis. Ann Intern Med. 2013; 159(4): 262-274.

20. Cherney DZ, Perkins BA, Soleymanlou N, et al. Renal hemodynamic effect of sodium-glucose cotransporter 2 inhibition in patients with type 1 diabetes mellitus. Circulation. 2014; 129(5): 587-597.

21. Abdul-Ghani M, Del Prato S, Chilton R, DeFronzo RA. SGLT2 Inhibitors and Cardiovascular Risk: Lessons Learned From the EMPA-REG OUTCOME Study. Diabetes Care. 2016; 39(5): 717-725.

22. Birkeland KI, Jørgensen ME, Carstensen B, et al. Cardiovascular mortality and morbidity in patients with type 2 diabetes following initiation of sodium-glucose co-transporter-2 inhibitors versus other glucoselowering drugs (CVD-REAL Nordic): a multinational observational analysis. Lancet Diabetes Endocrinol. 2017; 5(9): 709-717.

23. Usman MS, SiddiqiTJ, Memon MM, et al. Sodium-glucose co-transporter 2 inhibitors and cardiovascular outcomes: A systematic review and meta-analysis. Eur J Prev Cardiol. 2018; 25 (5): 495-502.

24. Kim Y, Babu AR. Clinical potential of sodium-glucose cotransporter 2 inhibitors in the management of type 2 diabetes. Diabetes Metab. Syndr. Obes. 2012; 5: 313-327.

25. Nisly SA, Kolanczyk DM, Walton AM. Canagliflozin, a new sodiumglucose cotransporter 2 inhibitor, in the treatment of diabetes. Am. J. Health Syst. Pharm. 2013; 70(4): 311-319.

26. Rosenstock J, Jelaska A, Frappin G, Salsali A, Kim G, Woerle HJ, Broedl UC. Improved glucose control with weight loss, lower insulin doses, and no increased hypoglycemia with empagliflozin added to titrated multiple daily injections of insulin in obese inadequately controlled type 2 diabetes. Diabetes Care. 2014 Jul; 37(7): 1815-1823.

27. Briand F, Mayoux E, Brousseau E, et al. Empagliflozin, via Switching Metabolism Toward Lipid Utilization, Moderately Increases LDL Cholesterol Levels Through Reduced LDL Catabolism. Diabetes. 2016; 65(7): 2032-2038.

28. Ferrannini G, Hach T, CroweS, et al. Energy Balance After Sodium-Glucose Cotransporter 2 Inhibition. Diabetes Care. 2015; 38(9): 1730-1735.

29. Amato MC, Giordano C, Galia M, et al. Visceral Adiposity Index: a reliable indicator of visceral fat function associated with cardiometabolic risk. Diabetes Care. 2010; 33(4): 920-922.

30. Zhao Y, Xu L, Tian D. Effects of sodium-glucose co-transporter 2 (SGLT2) inhibitors on serum uric acid level: A meta-analysis of randomized controlled trials. Diabetes Obes Metab. 2018; 20(2): 458-462.

31. Odden MC, Amadu AR, SmitE, et al. Uric acid levels, kidney function, and cardiovascular mortality in US adults: National Health and Nutrition Examination Survey (NHANES) 1988-1994 and 1999-2002. Am J Kidney Dis. 2014; 64(4): 550-557.

32. Feig DI, Kang DH, Johnson RJ. Uric acid and cardiovascular risk. N Engl J Med. 2008; 359(17): 1811-1821.

33. Vallon V. The mechanisms and therapeutic potential of SGLT- 2 inhibitors in diabetes mellitus. Annu. Rev. Med. 2015; 66: 255-270.

34. Scheen AJ, Delanaye P. Effects of reducing blood pressure on renal outcomes in patients with type 2 diabetes: Focus on SGLT2 inhibitors and EMPA-REG OUTCOME. Diabetes Metab. 2017; 43 (2): 99-109.

35. Borg R, Persson F. Empagliflozin reduces albuminuria-a promise for better cardiorenal protection from the EMPA-REG OUTCOME trial. Ann Transl Med. 2017; 5(23): 478.

36. Dekkers CCJ, Wheeler DC, Sjöström CD, Stefansson BV, Cain V, Heerspink HJL. Effects of the sodiumglucose co-transporter 2 inhibitor dapagliflozin in patients with type 2 diabetes and Stages $3 \mathrm{~b}-4$ chronic kidney disease. Nephrol Dial Transplant. 2018 Nov 1; 33(11): 2005-2011. 
37. Plutzky J, Bakris G. Sodium. Glucose Cotransporter 2 Inhibitors in Patients with Diabetes Mellitus and Chronic Kidney Disease. Circulation. 2018; 137: 130-133.

38. Shimizu M, Furuichi K, Toyama T, et al. Long-term outcomes of Japanese type 2 diabetic patients with biopsy-proven diabetic nephropathy. Diabetes Care. 2013; 36(11): 3655-3662.

39. Vallon V, Gerasimova M, Rose MA, et al. SGLT2 inhibitor empagliflozin reduces renal growth and albuminuria in proportion to hyperglycemia and prevents glomerular hyperfiltration in diabetic Akita mice. Am J Physiol Renal Physiol. 2014; 306(2): 194-204.

40. Ferrannini E, Ramos SJ, Salsali A, et al. Dapagliflozin monotherapy in type 2 diabetic patients with inadequate glycemic control by diet and exercise: a randomized, double-blind, placebo-controlled, phase 3 trial. Diabetes Care. 2010; 33(10): 2217-2224.

\section{ORCID and contributionship:}

Maryana M. Rosul - 0000-0002-2106-5386 A,B,D

Myroslava M. Bletskan -0000-0002-8069-6145 A,B,D

Natalia V. Ivanio - 0000-0003-0147-2176 ${ }^{\text {B,F }}$

Marina O. Korabelschykova - 0000-0002-7632-4322 ${ }^{B, F}$

Yelyzaveta I. Rubtsova - 0000-0001-9395-1822 E,F

\section{Conflict of interest:}

The Authors declare no conflict of interest.

\section{CORRESPONDING AUTHOR} Maryana M. Rosul

State Higher Educational Establishment "Uzhhorod National University", Uzhhorod, Ukraine

tel: +380506115733

e-mail: maryana.rosul@uzhnu.edu.ua

Received: 17.01 .2020

Accepted: 05.03 .2020

A - Work concept and design, B - Data collection and analysis, C - Responsibility for statistical analysis, D-Writing the article, $\mathbf{E}$-Critical review, $\mathbf{F}$ - Final approval of the article 